\title{
Intrinsically Disordered Region Modulates Ligand Binding in Glutaredoxin 1 from Trypanosoma Brucei
}

Galo. E. Balatti ${ }^{\mathrm{a}}$, G. Patricio Barletta ${ }^{\mathrm{a}}$, Gustavo Parisi ${ }^{\mathrm{a}}$, Silvio C. E. Tosatto ${ }^{\mathrm{b}}$, Massimo Bellanda ${ }^{\mathrm{c}}$, and Sebastian Fernandez-Albertia .

aDepartamento de Ciencia y Tecnologia, Universidad Nacional de Quilmes/CONICET, B1876BXD Bernal, Argentina.

${ }^{\text {b}}$ Department of Biomedical Sciences, University of Padova, Viale G. Colombo 3, 5131 Padua, Italy.

'Department of Chemical Sciences, University of Padova, via Marzolo 1, 35131, Padua, Italy.

\section{Supporting Information}

Figure S1. Normalized histograms of distances between the main pairwise residues that contribute to the stabilization of FL-1CGrx1 conformer \#1 (denoted in Figure 6(c)): A76D125, D50-T144, R133-D60, and R133-D53.

Figure S2. Normalized histograms of distances between the main pairwise residues that contribute to the stabilization of FL-1CGrx1 conformer \#2 (denoted in Figure 6(c)): V66A105, A71-A128, D69-K137, and D69-K96.

Figure S3. Normalized histograms of distances between the main pairwise residues that contribute to the stabilization of FL-1CGrx1 conformer \#3 (denoted in Figure 6(c)): I75V131.

All histograms display in Figures S1-S3 involve interactions between residues lining the surface of the binding pocket or contiguos to it and the intrinsically disordered N-terminal extension.

Figure S4. Normalized histograms of distances between K96-C104 that contribute to the stabilization of FL-1CGrx1 conformer \#3 compared with the corresponding histogram observed in $\Delta 76-1 C G r x 1$. 
Figure S5. Cumulative percentage of variance accounted for the $n^{\text {th }}$ lowest PCA modes during $5 \mu$ s of MD simulations for FL-1CGrx1 (red) and $\Delta 76-1 C G r x 1$ (blue).

Figure S6. Lower energy protein-ligand binding poses obtained by docking explorations performed on $(a, b)$ FL-1CGrx1 and (c,d) $\Delta 76$-1CGrx1 using original NMR structures. Glutathionylspermidine is used as ligand.

Figure S7. Structural superpositions between each FL-1CGrx1 conformer \#1 (yellow), \#2 (blue) and \#3 (green) and the $\Delta 76-1 \mathrm{CGrx} 1$ (black) obtained at the $\Delta \mathrm{G}$ basins indicated in Figure 6. The RMSFs of each FL-1CGrx1 conformer with respect to $\Delta 76-1 \mathrm{CGrx} 1$ are also depicted.

Figure S8. Evolution in time of projection of molecular dynamics simulations on the first and second Principal Component Analysis (PCA) vectors and the radius of gyration. Time intervals corresponding to each FL-1CGrx1 are indicated: conformer \#1 (yellow), \#2 (blue) and \#3 (green).

Figure S9. Matrix of the absolute values of the overlaps between PCA of FL-1CGrx1 and $\triangle 76-1 C G r x$. For the sake of comparison, the NTE tail of FL-1CGrx1 has not been considered in the PCA.

Table S1. List of residues lining the glutathione-binding pocket. 
Figure S1.
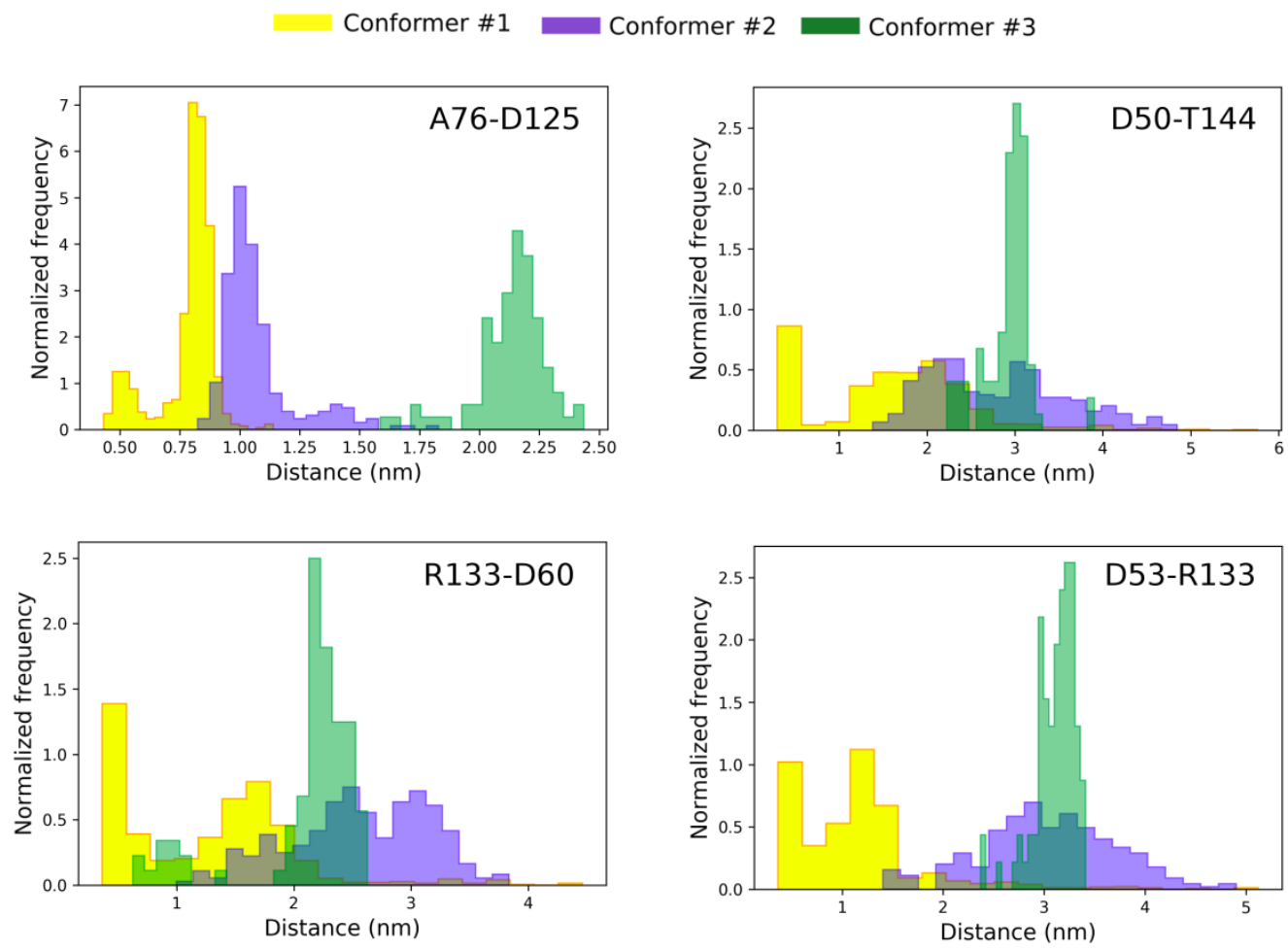
Figure S2.

Conformer \#1 Conformer \#2 Conformer \#3
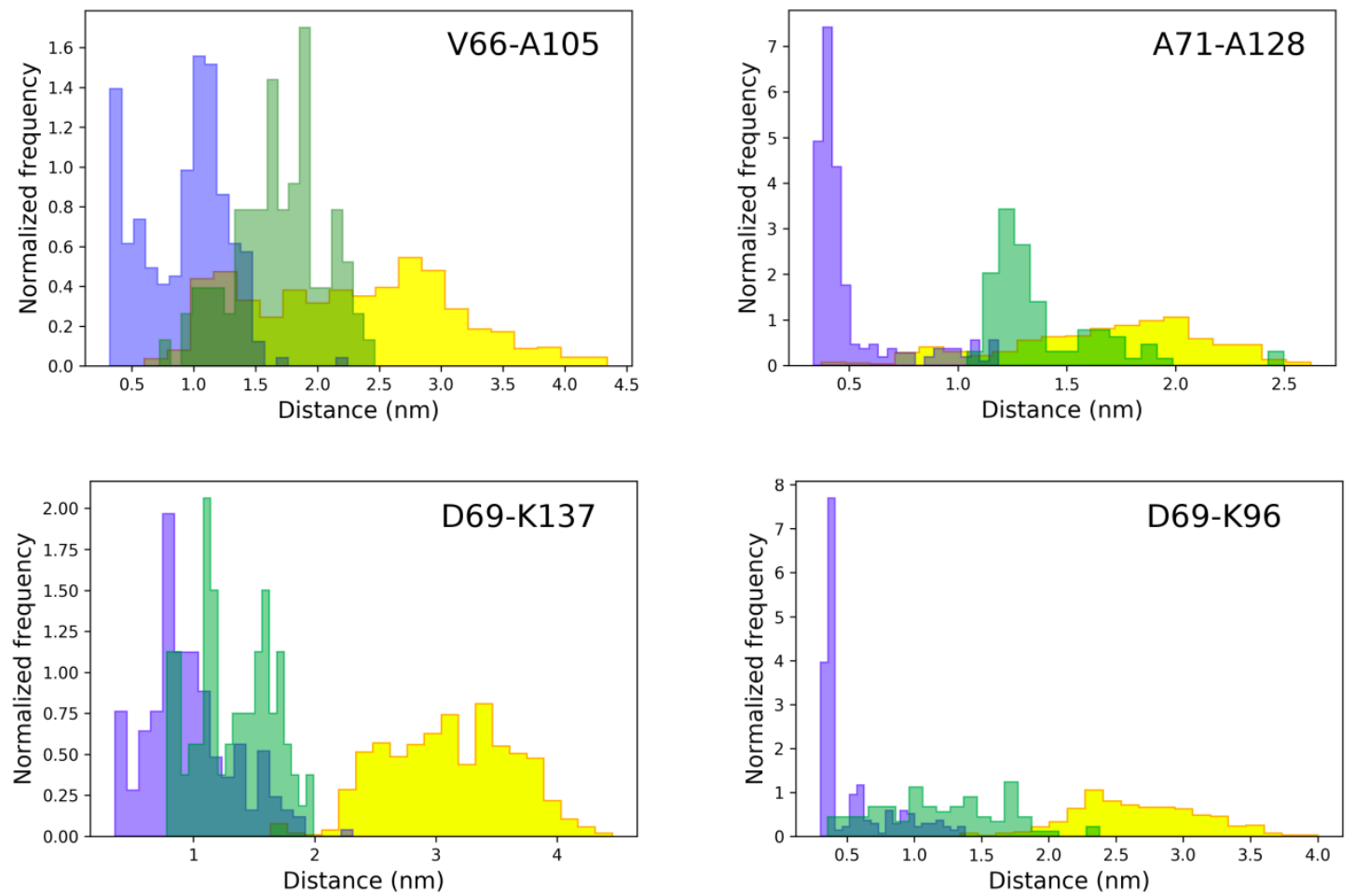
Figure S3.

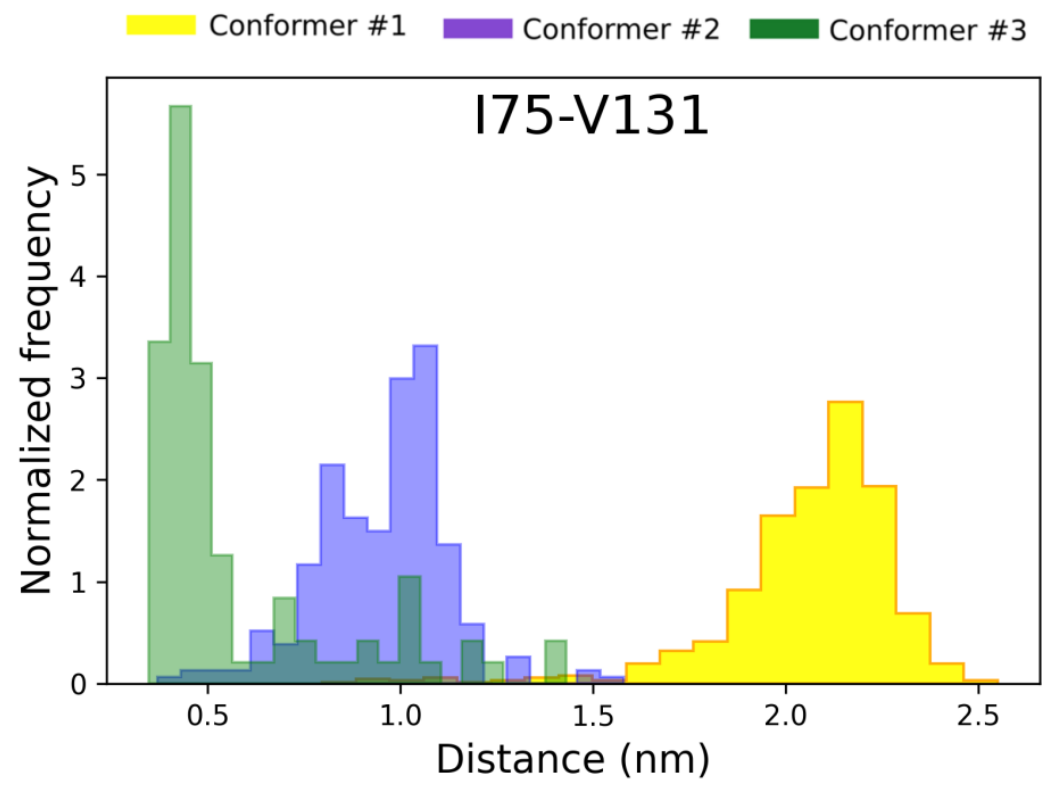


Figure S4.
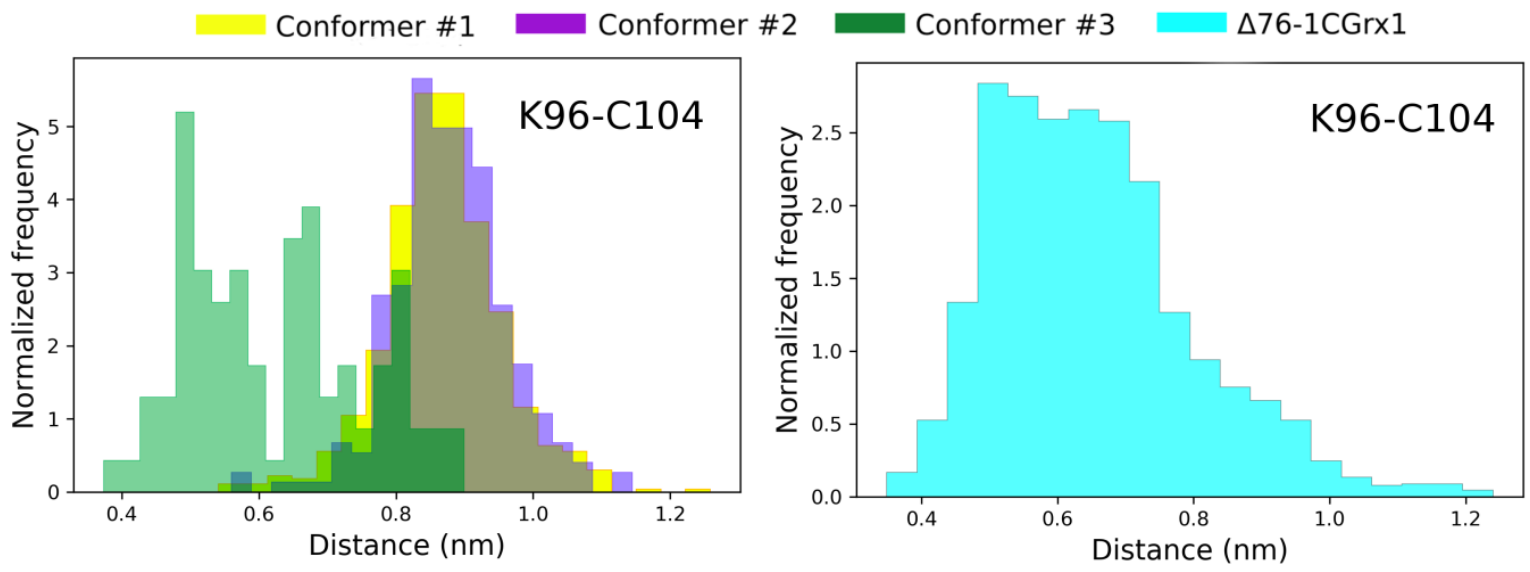
Figure S5.

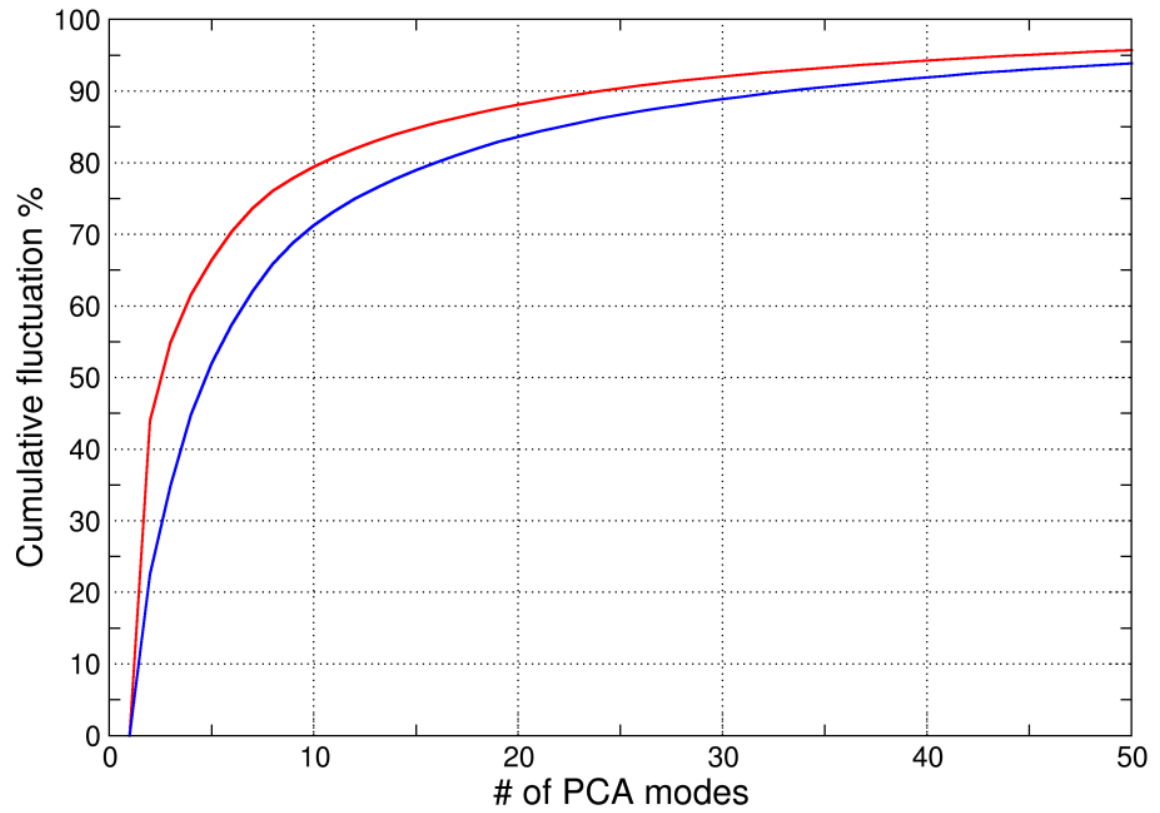


Figure S6.

(a)

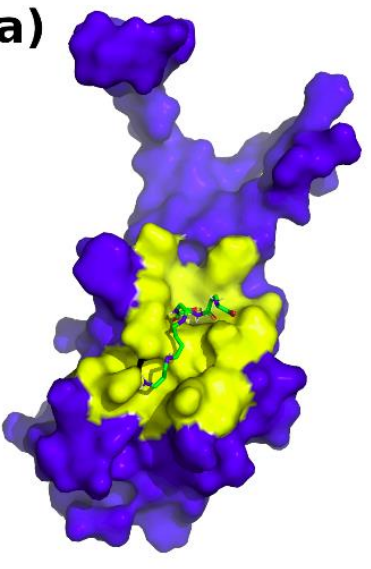

(c)

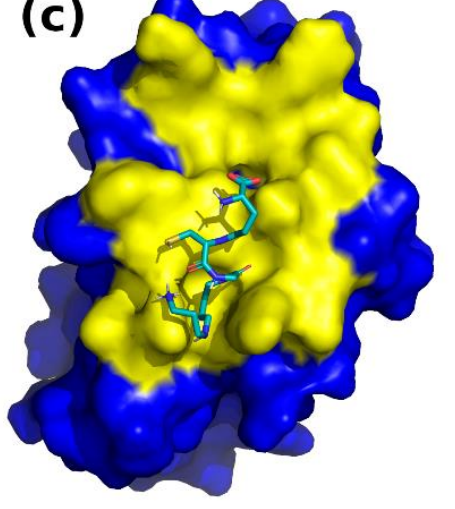

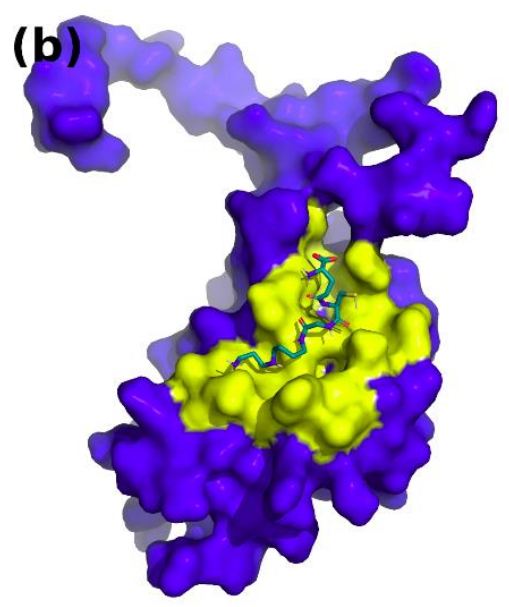

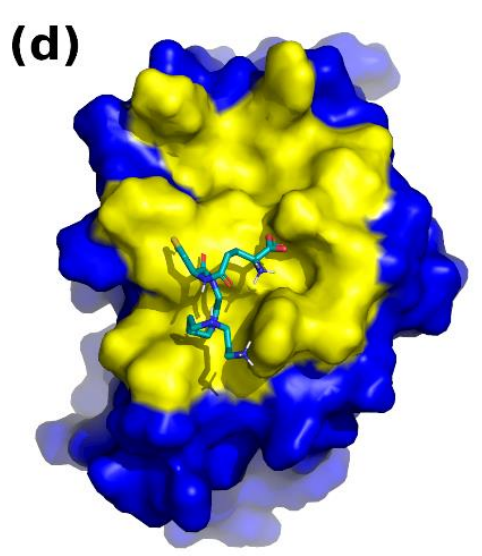


Figure S7.
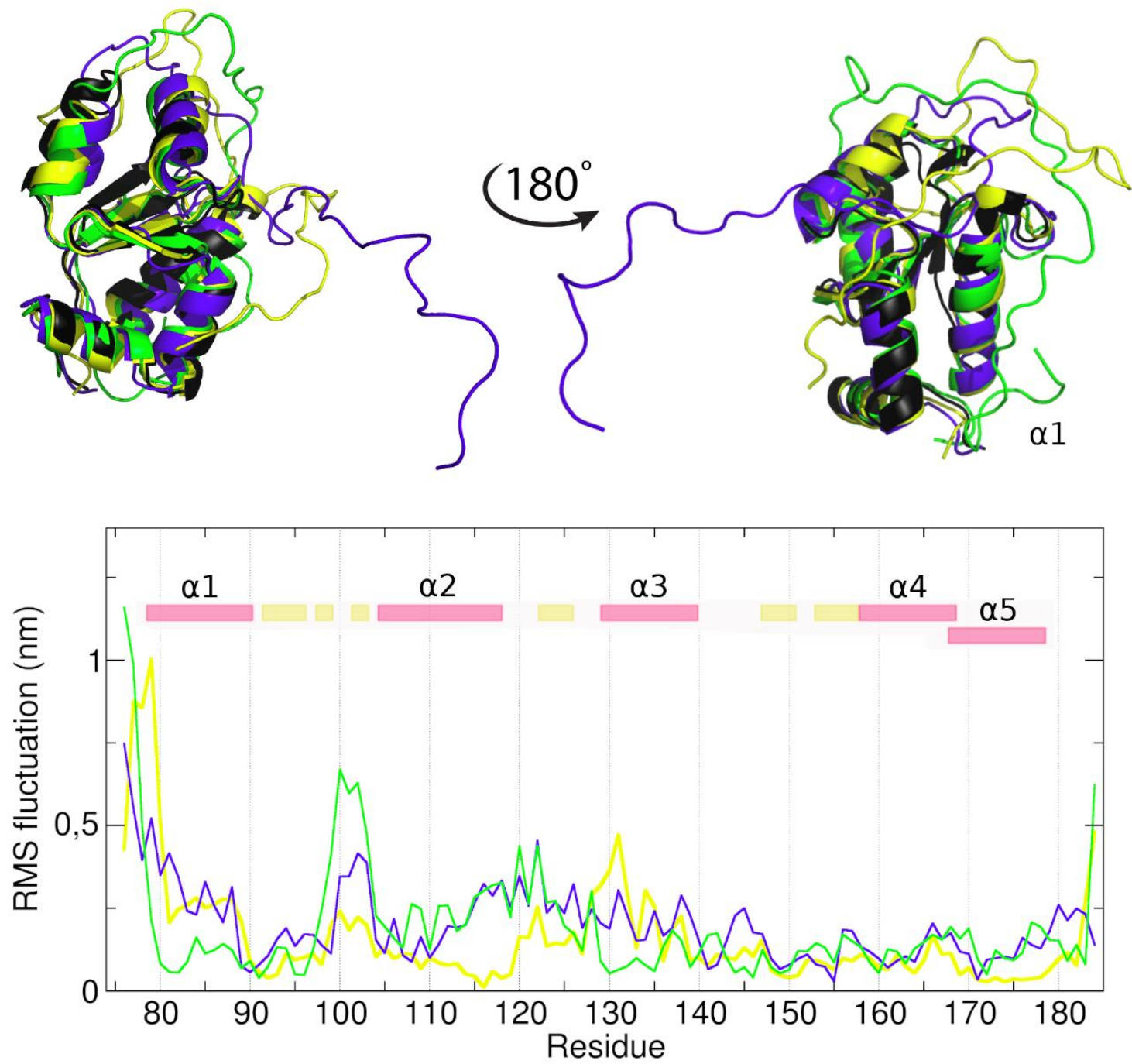
Figure S8.

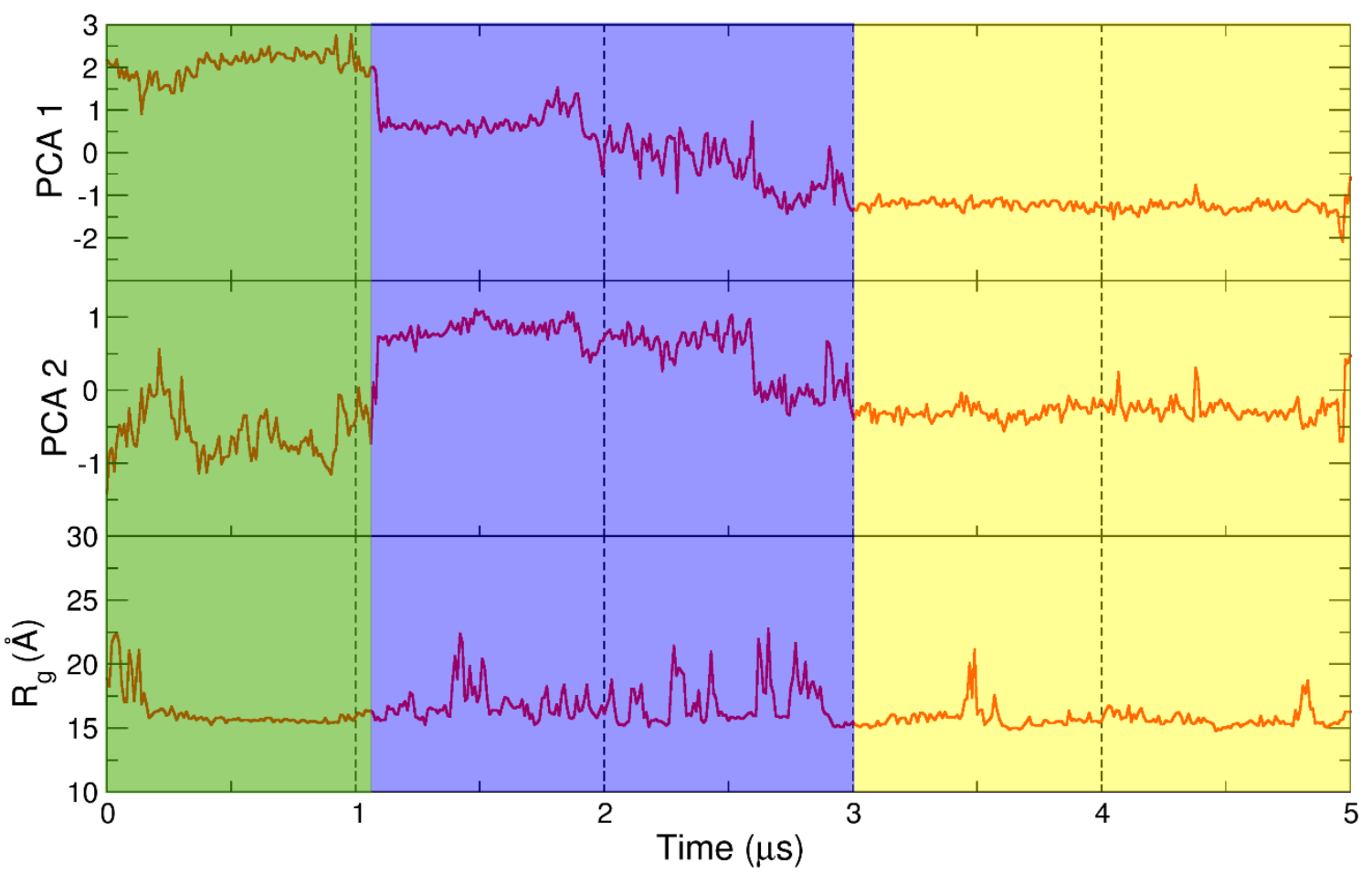


Figure S9.

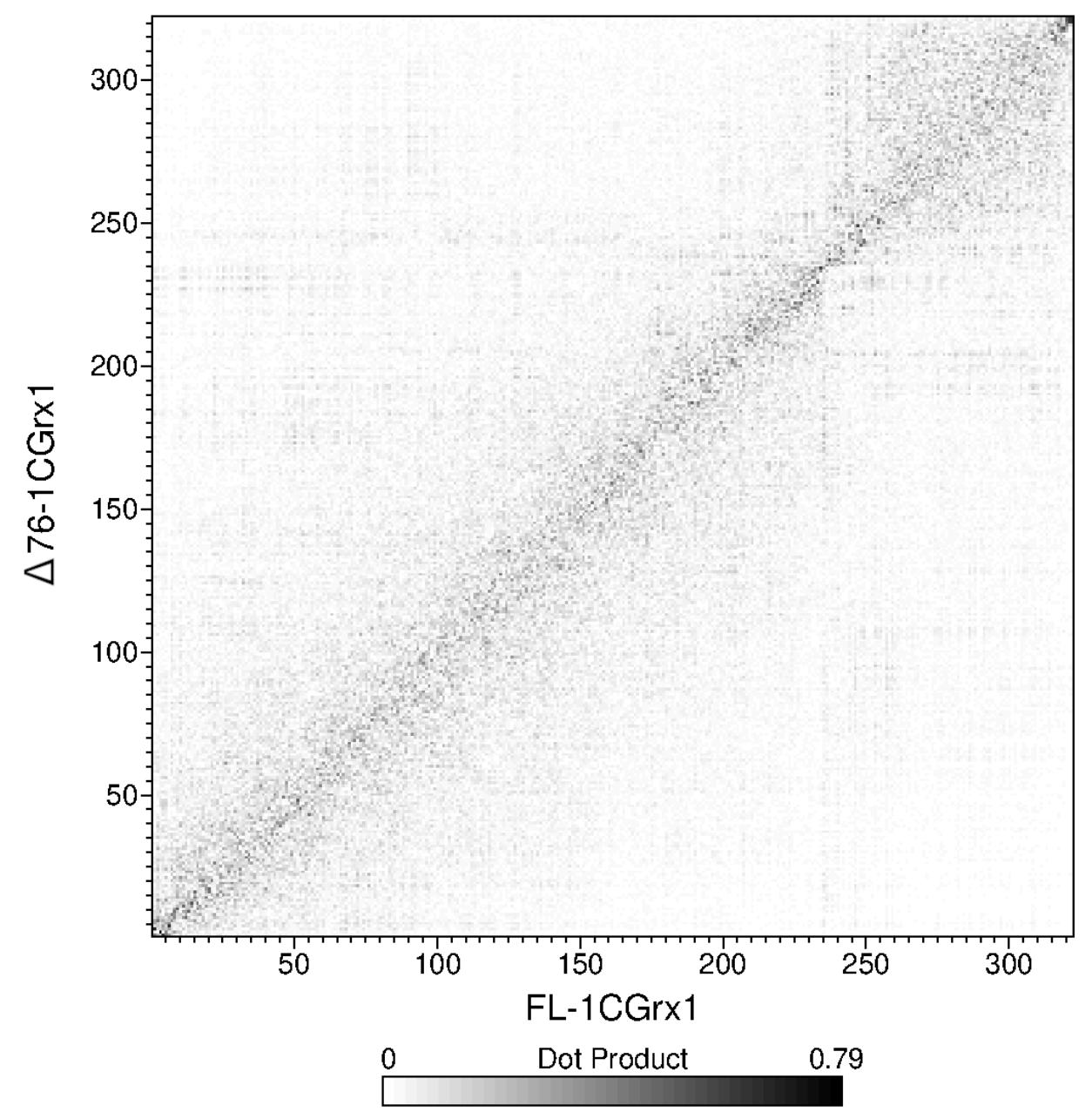


Table S1. List of residues lining the glutathione-binding pocket.

\begin{tabular}{|l|}
\hline Lys96 \\
\hline Cys104 \\
\hline Ala105 \\
\hline Tyr106 \\
\hline Ser107 \\
\hline Val126 \\
\hline Leu127 \\
\hline Arg133 \\
\hline Val136 \\
\hline Lys137 \\
\hline Pro143 \\
\hline Thr144 \\
\hline Iso145 \\
\hline Pro146 \\
\hline Gly157 \\
\hline Leu158 \\
\hline Asp159 \\
\hline Ile160 \\
\hline
\end{tabular}

